\title{
Mechanisms and Regulation of the Mitotic Inheritance of the Golgi Complex
}

\author{
Carmen Valente * and Antonino Colanzi * \\ Institute of Protein Biochemistry, National Research Council, Naples, Italy
}

In mammalian cells, the Golgi complex is structured in the form of a continuous membranous system composed of stacks connected by tubular bridges: the "Golgi ribbon." At the onset of mitosis, the Golgi complex undergoes a multi-step fragmentation process that is required for its correct partition into the dividing cells. Importantly, inhibition of Golgi disassembly results in cell-cycle arrest at the G2 stage, which indicates that accurate inheritance of the Golgi complex is monitored by a "Golgi mitotic checkpoint." Moreover, mitotic Golgi disassembly correlates with the release of a set of Golgi-localized proteins that acquire specific functions during mitosis, such as mitotic spindle formation and regulation of the spindle checkpoint. Most of these events are regulated by small

Edited by: Letizia Lanzetti, University of Turin, Italy

Reviewed by: Mitsuo Tagaya,

Tokyo University of Pharmacy and Life Sciences, Japan Charles Andrew Yeaman, University of lowa Carver College of Medicine, USA

*Correspondence: Carmen Valente c.valente@ibp.cnr.it; Antonino Colanzi a.colanzi@ibp.cnr.it

Specialty section

This article was submitted to Membrane Traffic,

a section of the journal Frontiers in Cell and Developmental

Biology

Received: 07 October 2015 Accepted: 27 November 2015 Published: 16 December 2015

Citation: Valente $C$ and Colanzi A (2015) Mechanisms and Regulation of the Mitotic Inheritance of the Golgi Complex. Front. Cell Dev. Biol. 3:79. doi: 10.3389/fcell.2015.00079 GTPases of the Arf and Rab families. Here, we review recent studies that are revealing the fundamental mechanisms, the molecular players, and the biological significance of mitotic inheritance of the Golgi complex in mammalian cells. We also briefly comment on how Golgi partitioning is coordinated with mitotic progression.

Keywords: cell cycle, mitosis, golgi complex, mitotic spindle, Arf, Rab

\section{INTRODUCTION}

The Golgi complex is the central organelle in the secretory pathway, and it mediates the modification, sorting and transport of proteins and lipids (De Matteis and Luini, 2008). The structural organization of the Golgi complex varies across organisms. In mammals, it consists of flat cisternae that are grouped into several stacks (the compact zones) that are themselves laterally interconnected by tubular membrane "bridges" (the non-compact zones) to form the "Golgi ribbon" (Sütterlin and Colanzi, 2010). The complex organization of the Golgi ribbon is highly dynamic, and this allows large rates of membrane flux and rapid changes in shape, combined with disassembly and reassembly of the Golgi ribbon under different physiological conditions (Rothman and Wieland, 1996).

For instance, during cell division, these Golgi membranes must be exactly partitioned into the two daughter cells, as for the other organelles and the DNA. This Golgi segregation occurs through regulated and reversible multi-step ribbon disassembly, to produce small fragments that can then be inherited by the daughter cells. This process initiates in the G2 phase of the cell cycle, when the Golgi ribbon is cut into individual Golgi stacks. Then during prophase/prometaphase, these

Abbreviations: Arf, ADP-ribosylation factor; Cdk1, Cyclin dependent kinase 1; CtBP1-S/BARS, C-terminal-binding protein 1, short form/brefeldin-A-dependent ADP-ribosylation substrate; ERK1, extracellular-signal-regulated kinase 1; GRASP, Golgi complex reassembly stacking protein; MEK1, mitogen-activated protein kinase kinase 1; Plk, Polo-like kinase; Rab, Ras-related GTP-binding protein; Raf1, recombinase-activating factor 1; VRK1, vaccinia related kinase 1. 
are unstacked and undergo vesiculation, followed by progressive shortening into vesicular/tubular clusters and small fragments. At metaphase, these fragments appear as the "Golgi haze." At telophase, this haze is gradually reassembled into stacks, to eventually re-form a Golgi ribbon in each of the daughter cells (Shorter and Warren, 2002; Altan-Bonnet et al., 2004; Colanzi and Corda, 2007).

Inhibition of Golgi ribbon unlinking induces a potent and persistent block in the G2 phase of cell-cycle progression (Sütterlin et al., 2002; Hidalgo Carcedo et al., 2004), which is known as the "Golgi checkpoint." This novel cell-cycle checkpoint is not mediated by activation of the DNA-damage checkpoint; instead, it has been postulated to sense the integrity of the Golgi complex (Sütterlin et al., 2002; Hidalgo Carcedo et al., 2004). Thus, these studies have revealed that Golgi partitioning is an essential and required event for cell entry into mitosis. This highlights the regulatory interplay between organelle inheritance and the signaling pathways that regulate cell division.

Here, we review the current understanding of the mechanisms of Golgi ribbon breakdown in mammalian cells in G2, and we discuss the signaling events that coordinate this Golgi checkpoint.

\section{ORGANIZATION OF MAMMALIAN GOLGI MEMBRANES IN INTERPHASE}

The number of cisternae within a Golgi stack and the number of Golgi stacks in a cell are extremely variable across different species and tissues. In the yeast Saccharomyces cerevisiae there is no stacked Golgi structure, and instead the Golgi exists as individual cisternae that are dispersed throughout the cytoplasm (Rossanese et al., 1999). In contrast, in the yeast Pichia pastoris, each Golgi stack contains three or four cisternae (Mogelsvang et al., 2003). Up to 30 flattened cisternae per stack have been observed in plants and lower organisms, while mammalian cells can have up to 100 Golgi stacks, each composed of 5-8 cisternae (Beams and Kessel, 1968).

The characteristic structure of the Golgi stack and its ribbon organization in mammals rely on numerous molecular machineries. Among these, the peripheral membrane golgins and the Golgi matrix proteins (i.e., GRASP65, GRASP55) mediate the functional three-dimensional arrangement of the Golgi membranes (Puthenveedu et al., 2006; Feinstein and Linstedt, 2008; Munro, 2011). The Golgi ribbon organization is also under the control of an intact actin and microtubule cytoskeleton, as well as specialized cytoskeleton-based motors and membrane input from the endoplasmic reticulum (ER; Thyberg and Moskalewski, 1999; Rios and Bornens, 2003; Marra et al., 2007). The Golgi complex is also positioned in a perinuclear region near to the centrosome, where it remains due to a microtubule-dependent mechanism (Rambourg and Clermont, 1990).

However, why the Golgi ribbon is organized into stacks of cisternae in interphase remains an important unresolved issue. It is indeed questionable whether cisterna stacking is required for protein transport, particularly considering the efficient secretion in S. cerevisiae where the Golgi cisternae are unstacked and dispersed throughout the cytoplasm (Papanikou and Glick, 2009).

Although, the functional significance of the pericentriolar position of Golgi stacks is not completely understood, it has been recently proposed to contribute to: (i) the interactions between the Golgi complex and the centrosome, to facilitate Golgi-mediated centrosome organization and function (Kodani and Sütterlin, 2009); (ii) cell entry into mitosis after Golgi ribbon fragmentation and temporary loss of its pericentriolar position (Sütterlin et al., 2002); and (iii) cell polarization and migration. For example, disruption of the pericentriolar position after Golgin-160 or GMAP210 depletion affects directional protein secretion, which is required for cell migration (Yadav et al., 2009). In addition, increased protein transport rate and glycosylation defects have been shown recently as a result of loss of Golgi stacking (Xiang et al., 2013). This thus, indicates a role for cisterna stacking in the regulation of the accuracy of protein glycosylation.

In conclusion, the ribbon organization, cisterna stacking, and pericentriolar position of the Golgi complex appear to add another level of regulation for these mammalian-cell-specific processes.

\section{MECHANISM OF MITOTIC GOLGI DISASSEMBLY}

Mitotic segregation of the Golgi complex involves progressive and reversible disassembly of the Golgi ribbon into dispersed fragments (Figure 1), to allow correct partitioning of the Golgi membranes between daughter cells (Shorter and Warren, 2002; Colanzi et al., 2003; Altan-Bonnet et al., 2004). A number of the molecular players here have been identified and characterized through two biochemical approaches: semi-intact cell assays, and Golgi mitotic disassembly/reassembly assays (Tang et al., 2010; Colanzi and Sütterlin, 2013). These two assays have provided a highly manipulable approach, within which the sequence of morphological events can be precisely followed by electron microscopy or biochemical analysis.

\section{A. Severing the Golgi Ribbon into Stacks}

The first step of mitotic Golgi disassembly occurs in the G2 phase and consists of the fragmentation of the non-compact zones of the Golgi ribbon. Then, at the onset of mitosis, these isolated Golgi stacks are converted into scattered tubulereticular elements that are further fragmented and dispersed throughout the cytoplasm (Figure 1). The cleavage of these membrane tubules is now known to be necessary and sufficient for cell entry into mitosis (Colanzi et al., 2007; Feinstein and Linstedt, 2007). Several crucial factors involved in this mitotic Golgi disassembly have been identified. Among these, there are the fission-inducing protein CtBP1-S/BARS (referred to here as BARS; Hidalgo Carcedo et al., 2004) and the peripheral Golgi proteins GRASP65 and GRASP55 (Sütterlin et al., 2002; Xiang and Wang, 2010). Each of these proteins has a specialized role in Golgi-ribbon unlinking, and their combined activities 


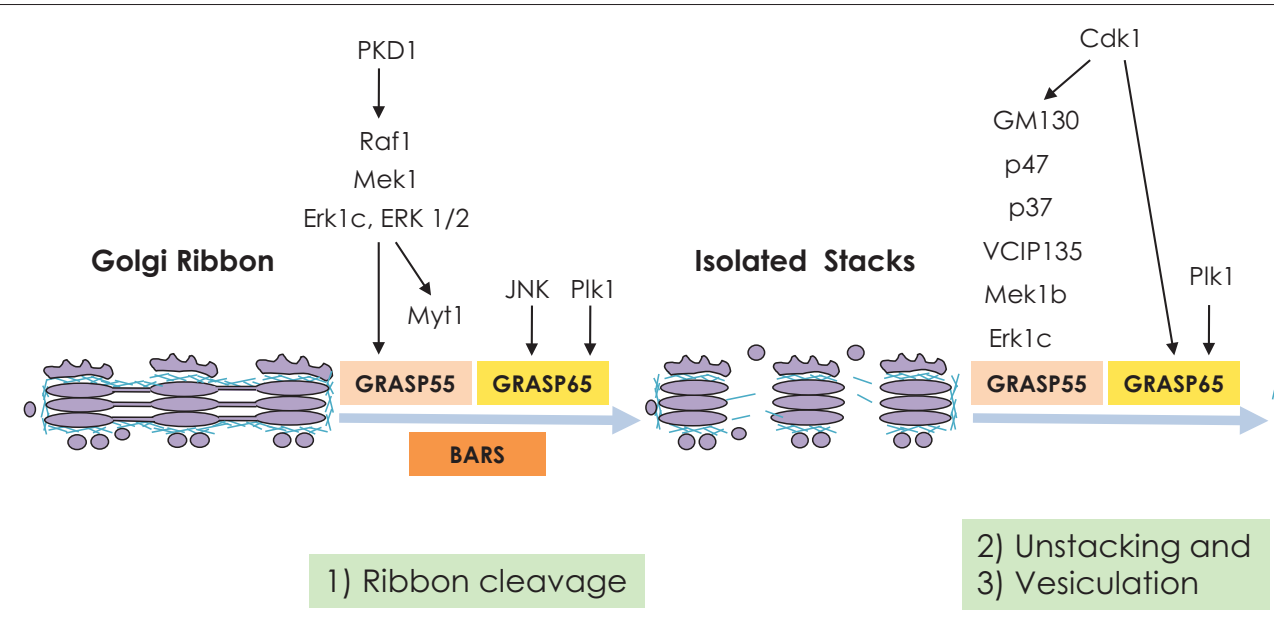

FIGURE 1 | Schematic representation of mitotic partitioning of the Golgi ribbon. This first step of mitotic Golgi fragmentation (i.e., ribbon unlinking) controls G2/M transition and requires the activities of BARS, GRASP55, and GRASP65. These processes also require the activities of several MAP kinase components, as indicated. At the onset of mitosis, these isolated stacks undergo further disassembly in the sequential steps of unstacking and vesiculation. These lead to the formation of the so-called "Golgi haze" during metaphase, where the Golgi membranes are completely fragmented. These processes require the activities of the kinases Plk1 and Cdc2, and their targets GRASP55 and GRASP65. Subsequent events result in dephosphorylation of the kinases that took part in the initial fragmentation, which leads to reassembly of the Golgi ribbon in the daughter cells.

drive the cleavage of the Golgi interstack connections in late G2 phase of the cell cycle. Roles for these proteins in the further fragmentation of the isolated Golgi stacks into the "Golgi haze" that is detected in metaphase have also been shown (Figure 1).

\section{BARS}

BARS is a protein with a dual role, as it can act as a transcriptional co-repressor in the nucleus, and as a regulator of Golgi tubule fission in membrane trafficking (Hidalgo Carcedo et al., 2004; Colanzi et al., 2007). BARS controls mitotic disassembly of the Golgi stacks by severing the tubular network of the non-compact zones (Colanzi et al., 2007). The functional role of BARS in mitosis has been studied both in vitro and in vivo. In vitro, both the depletion and inhibition of BARS strongly inhibit mitotic Golgi fragmentation. In-vivo studies using fluorescence recovery after photobleaching have defined the role of BARS in the fission of the interstack connecting tubules, which is a crucial step for G2/M cell-cycle transition (Colanzi et al., 2007). However, interference with BARS activity in interphase does not lead to Golgi fragmentation (Hidalgo Carcedo et al., 2004), which indicates that BARS is specifically activated in G2 phase to promote the severing of the Golgi ribbon. It is reasonable that this activation is mediated by a specific BARS phosphorylation event that occurs in the G2/M phases, as has been shown for BARS activation in membrane traffic (Liberali et al., 2008; Valente et al., 2012, 2013). BARS phosphorylation might induce its binding to specific partners that then promote Golgi ribbon fission. Moreover, BARSknockout fibroblasts have their Golgi ribbon divided into isolated stacks at all cell-cycle stages, which bypasses the requirement for BARS for G2/M Golgi ribbon unlinking (Colanzi et al., 2007).

\section{Golgi Reassembly and Stacking Proteins (GRASPs)}

The mitotic functions of the Golgi reassembly stacking proteins (hence GRASPs) GRASP65 and the structurally related GRASP55 are better defined. Both GRASP65 and GRASP55 are peripheral membrane proteins that have two N-terminal PDZ-like domains and a C-terminal serine/proline-rich regulatory domain (Vinke et al., 2011). The localization of GRASP65 to the cis-Golgi and of GRASP55 to the medial/trans Golgi is mediated by their Nterminal myristic acid (the GRASP domain) and their binding to specific golgins (Vinke et al., 2011). Both GRASP65 and GRASP55 are essential factors for formation and maintenance of the tubules that connect the Golgi stacks (Sütterlin et al., 2005; Puthenveedu et al., 2006; Feinstein and Linstedt, 2008). Depletion of GRASP65 and GRASP55 induces Golgi ribbon unlinking and a reduction in the number of cisternae per stack (Sütterlin et al., 2005; Puthenveedu et al., 2006; Feinstein and Linstedt, 2008; Jarvela and Linstedt, 2014). Moreover, inhibition of the activities of GRASP65 and GRASP55 alters mitotic Golgi fragmentation and mitotic entry (Sütterlin et al., 2002; Preisinger et al., 2005; Duran et al., 2008).

\section{GRASP65}

GRASP65 is phosphorylated at multiple sites in its C-terminal serine/proline-rich domain during mitosis, by Cdk1 and Plk1 kinases (Lin et al., 2000; Wang et al., 2003; Preisinger et al., 2005; Sütterlin et al., 2005; Yoshimura et al., 2005). In-vitro studies have shown that the GRASP proteins form homo-oligomers in trans, and these interactions are prevented by phosphorylation of specific residues within the C-terminal GRASP65 serine/prolinerich domain (Tang et al., 2010). Cdk1 targets GRASP65 at four serine/threonine residues (i.e., Ser216/Ser217, Thr220, Ser277, Ser376). Accordingly, mutation of these sites into alanines prevents mitotic Golgi unlinking. Plk1 has been shown to dock 
onto Cdk1-phosphorylated GRASP65 and then to phosphorylate GRASP65 at Ser189. This Cdk1-mediated and Plk1-mediated phosphorylation abrogates the trans-oligomerization properties of GRASP65. The consequence of this is prevention of its tethering function for the connection of the cisternae in the formation of the stacked Golgi, and its linking of adjacent stacks to form the Golgi ribbon (Wang et al., 2003, 2005; Sengupta and Linstedt, 2010). More recently, it has been shown that the kinase JNK2 is a potent and crucial regulator of Golgi unlinking during G2. This action of JNK2 is mediated by phosphorylation of Ser277 of GRASP65, which reveals a JNK2GRASP65 signaling axis that couples Golgi inheritance and G2/M transition (Cervigni et al., 2015). Altogether, these data suggest a link between the C-terminus phosphorylation events on GRASP65 and mitotic Golgi fragmentation. This hypothesis has been recently validated by studies of the conformational changes in the crystal structure of the GRASP65-PDZ domain under Plk1mediated phosphorylation, which alters the membrane tethering function of GRASP65 (Truschel et al., 2012). Moreover, injection of an affinity-purified blocking anti-GRASP65 antibody (which recognizes its C-terminal region) into mitotic cells inhibits the reforming of the stacks and of the Golgi ribbon after cell division (Wang et al., 2003).

\section{GRASP55}

Similar to GRASP65, GRASP55 is phosphorylated during mitosis. ERK2 is a downstream target of mitogen-activated protein (MAP) kinase kinase 1 (MEK1) and it phosphorylates GRASP55, which has been shown to be required for Golgi ribbon unlinking and mitotic progression (Duran et al., 2008; Feinstein and Linstedt, 2008; Xiang and Wang, 2010). Of note, expression of a MEK1/ERK2-mediated phospho-defective mutant of GRASP55 (T222, 225A) prevents Golgi fragmentation and the consequent G2/M cell-cycle transition (Feinstein and Linstedt, 2008). This MEK1/ERK2/GRASP55-mediated G2/M block/delay is rescued in GRASP65-depleted cells where the Golgi ribbon is already fragmented (Feinstein and Linstedt, 2008). These data indicate that GRASP65 and GRASP55 cooperate in Golgi fragmentation and mitotic progression.

\section{Key Regulatory Pathways PKD/RAF/MEK/ERK/GRASP55}

The RAF1/MEK/ERK signaling pathway is the best characterized kinase cascade in G2/M unlinking of the Golgi ribbon (Acharya et al., 1998; Feinstein and Linstedt, 2007). MEK1 is first mitotically activated by RAF1, and then recruited to the Golgi ribbon in late prophase, where it mediates Golgi complex fragmentation (Colanzi et al., 2003). Accordingly, blocking MEK1 function by RNA interference (RNAi) or using the MEK1/2-specific inhibitor U0126 prevents ribbon unlinking, which inhibits cells entry into mitosis (Feinstein and Linstedt, 2007). The activation of RAF1/MEK1 in mitosis is also under the control of PKD1 and PKD2 (Kienzle et al., 2013), which are key factors in the regulation of post-Golgi carrier formation (Yeaman et al., 2004; Valente et al., 2012). Interfering with PKD activity blocks mitotic activation of RAF1 and MEK1, and, as a consequence, ribbon unlinking is blocked, and the cells accumulate in the G2 phase (Kienzle et al., 2013). Of note, this phenotype is recovered by expression of active MEK1, which indicates that PKD is involved in the same RAF1/MEK1 regulatory Golgi partitioning pathway (Kienzle et al., 2013). Among the downstream MEK1 substrates, the best characterized is ERK1/2, which phosphorylates GRASP55 to induce mitotic Golgi breakdown (Feinstein and Linstedt, 2008). ERK1c is the second MEK1 effector, which is a splice variant of ERK1 that is highly expressed, phosphorylated and activated in mitosis. ERK1c is activated by MEK1b-mediated phosphorylation. Modulation of ERK1c endogenous levels strongly affects mitotic Golgi fragmentation (i.e., overexpression, increased fragmentation; depletion, impaired fragmentation; Shaul and Seger, 2006). In addition, Plk3 (Polo like kinase 3) has been identified as another Golgi-localized downstream target of MEK1/ERK kinases (Ruan et al., 2004). Plk3 activation induces Golgi ribbon fragmentation (Ruan et al., 2004), and in embryonic fibroblasts from Plk3 knock-out mice, this fragmentation is partially inhibited (Xie et al., 2004). Furthermore, Plk3 binds, phosphorylates and activates VRK1. Inhibition of VRK1 function hampers MEK1-induced Golgi fragmentation (López-Sánchez et al., 2009). However, for these Plk3, VRK1, and ERK1c actions in the severing of the Golgi ribbon and in the conversion of mini-stacks into "Golgi blobs," their specific contributions and Golgi targets remain unknown. Finally, the protein kinase Myt1 is an important MEK effector, as it negatively regulates Cdk1 activity and is associated with the Golgi complex and the ER (Liu et al., 1997). RNAi studies in Drosophila and HeLa cells have revealed a requirement for Myt1 in mitotic Golgi dynamics (Cornwell et al., 2002; Nakajima et al., 2008). In addition, a recent study reported that Mytl depletion causes accelerated entry into mitosis and increased fragmentation of the Golgi complex during G2 (Villeneuve et al., 2013). The hypothesis that Myt1 functions downstream of MEK1 was suggested by the finding that Myt1 is inactivated by MEK1-mediated phosphorylation, and that Myt1 depletion bypasses the requirement for MEK1.

\section{PIk1/GRASP65 and JNK2/GRASP65 Pathways}

Plk1 is one of the key actors in mitotic Golgi fragmentation (Sütterlin et al., 2001; Sengupta and Linstedt, 2010). Plk1 phosphorylates GRASP65 on Ser189, which is part of the $\mathrm{N}$-terminal domain that mediates trans-oligomerization of GRASP65 (Truschel et al., 2012). Mutation of this residue to aspartic acid inhibits the GRASP65-mediated tethering function, while alanine substitution hampers mitotic Golgi unlinking (Sengupta and Linstedt, 2010). A model can be hypothesized in which the C-terminal domain of GRASP65 is first phosphorylated by Cdk1, to create a Plk1 binding site. Then GRASP65 is phosphorylated by Plk1 on Ser189, which inhibits the trans-oligomerization of GRASP65. However, as the activity of Cdk1 in G2 is low, it is possible to speculate that in addition to Cdk1, other kinases might phosphorylate GRASP65 to generate a Plk1 binding side. Moreover, it has been recently shown that during the G2 phase, GRASP65 is specifically phosphorylated in Ser227 by JNK2 kinase (Cervigni et al., 2015). The blocking of JNK2 kinase function by RNAi or using three unrelated JNK inhibitors persistently blocks the cell cycle in G2 phase. This 
study led to a model in which the JNK2/GRASP65 pathway is a crucial linker of mitotic Golgi ribbon partitioning with G2/M phase transition (Cervigni et al., 2015).

\section{B. Disassembly of Golgi Stacks into "Blobs" and "Haze"}

The further fragmentation of the isolated Golgi stacks starts in prophase and results in dispersed "Golgi blobs" and "Golgi haze" throughout the cytoplasm (Misteli and Warren, 1995). This additional fragmentation step depends on the unstacking of the Golgi membranes, followed by an additional vesiculation step. Golgi cisterna unstacking requires Cdk1mediated phosphorylation of GRASP65 and GRASP55, and thereby inhibition of their formation of trans-oligomers. In cell-free systems, mitotic disassembly of Golgi cisternae occurs concomitant to unstacking, and can follow two distinct pathways. One pathway proceeds via COPI vesicles, which continue to bud from the Golgi stack (Sönnichsen et al., 1996) without tethering to their target membrane (Shorter and Warren, 2002). Concomitantly, a COPI-independent pathway breaks up the flattened cisternae into tubular-vesicular fragments (Shorter and Warren, 2002). The nature of these fragments and the mechanisms of their inheritance in the two daughter cells have been described according to two opposing models.

The first model suggests that the Golgi complex is in dynamic equilibrium with the ER, and thus a block of membrane transport in mitosis would induce Golgi membrane disassembly (Zaal et al., 1999; Altan-Bonnet et al., 2004; Rhee et al., 2005). The inactivation of two small GTPases that operate at the ER/Golgi interface, Arf1 and Sar1, would mediate this effect (Altan-Bonnet et al., 2004). In prophase, Cdc2-mediated inactivation of Sar1 would reduce the export of membranes and proteins from the ER (Prescott et al., 2001; Kano et al., 2004). As a consequence, the membrane transport cycle between the Golgi complex and the ER would be impaired, which would induce progressive redistribution of Golgi membranes into the ER. The rate of this accumulation would be further enhanced by increased protein transport from the Golgi to the ER in the initial phases of mitosis, after dissociation of Arf1 from the Golgi membranes (AltanBonnet et al., 2004). This model finally suggests that the Golgi is inherited together with the ER (Zaal et al., 1999), and at mitotic exit, the restarting of membrane transport would re-establish normal interphase Golgi structure and function (Altan-Bonnet et al., 2004).

The second model suggests that the mechanism of Golgi inheritance is achieved in an ER-independent manner. Thus, it indicates that the Golgi complex is an autonomous organelle that forms the mitotic Golgi remnants required for postmitotic reassembly (Barr, 2004). In this case, the key event of Golgi inheritance is the disruption of the membrane tethering complexes by mitotic-activated kinases (Shorter and Warren, 2002). As a result, the Golgi stacks are fragmented into tubularvesicular clusters and the small vesicles of the Golgi haze, which are then actively partitioned between the two daughter cells in a mitotic-spindle-mediated process (Shima et al., 1998; Seemann et al., 2002; Axelsson and Warren, 2004; Barr, 2004; Puri et al., 2004). According to this model, at the end of mitosis, inhibition of mitotic kinases would reverse this series of events to allow the Golgi to be reassembled.

Many pieces of evidence support the second ER-independent Golgi partitioning model. Indeed, it has been shown that mitotic Golgi fragments accumulate near the spindle poles, while the ER is not present in the spindle area (Puri et al., 2004). Additionally, Seemann and co-workers provided evidence in favor of spindledependent Golgi ribbon inheritance (Wei and Seemann, 2009). Indeed, through induction of asymmetric cell division where the spindle was inherited by only one of the daughter cells, they demonstrated that the Golgi reassembled into a ribbon only in the cell with the inherited spindle. Conversely, in the spindledeprived cell, the Golgi stacks remained dispersed throughout the cytoplasm (Wei and Seemann, 2009). Interestingly, the injection of Golgi protein extracts into these spindle-deprived cells with the addition of purified spindle proteins promoted the reforming of an intact Golgi ribbon (Wei and Seemann, 2009). Thus, the accuracy of Golgi inheritance suggests the involvement of an active and regulated partitioning mechanism, instead of a stochastic process (Shima et al., 1998).

\section{MOLECULAR MECHANISM OF POST-MITOTIC GOLGI REASSEMBLY}

In-vitro cisternal regrowth assays from mitotic Golgi fragments have identified NSF (N-ethylmaleimide-sensitive factor) and p97 as two key factors in post-mitotic Golgi reassembly (Acharya et al., 1995; Rabouille et al., 1995). NSF and p97, which is an NEM-sensitive protein homologous to NSF (and is also known as VCP; valosin-containing protein), are ATPases that are associated with a variety of cellular activities of the AAA family of proteins (Brunger and DeLaBarre, 2003). As ATPases, NSF and p97 have specific co-factors: NSF-mediated fusion requires the proteins SNAPs and $\mathrm{p} 115$, while the p97-mediated pathway requires formation of a complex with p47 and VCIP135, or with p37 (Kondo et al., 1997; Uchiyama et al., 2006). In the post-mitotic Golgi-reassembly process, two sequential events have been suggested: first, membrane fusion induced by NSF produces large vesicles and tubular-reticular elements, which then fuse to generate cisternae through a p97-mediated process. During mitosis, Cdk1 phosphorylates p47, p37 and VCIP135, and blocks p97-controlled membrane-fusion processes so that the Golgi membranes remain disassembled (Uchiyama et al., 2003; Kaneko et al., 2010; Totsukawa et al., 2013). Thus, mitotic phosphorylation of the membrane fusion machinery can explain the mitotic Golgi phenotype of dispersed tubularreticular membranes and vesicles in the cytosol.

Additionally, ubiquitination has been shown to have an important role in post-mitotic Golgi reassembly. P47, the adaptor protein of $\mathrm{p} 97$, contains a UBA ubiquitin binding domain. P97 binding promotes $\mathrm{p} 47$ binding to ubiquitinated proteins via its UBA domain, and this domain is necessary for Golgi reassembly (Meyer et al., 2000). VCIP135 (valosin-containing protein p97/p47 complex-interacting protein; p135) is a positive factor for p97/p47-mediated membrane fusion (Uchiyama et al., 2002). Indeed, it has been shown to have a deubiquitinase activity, which 
was necessary for Golgi reassembly (Zhang and Wang, 2015). In early mitosis, Cdk1-mediated phosphorylation on Ser130 inhibits the deubiquitinase activity of VCIP135, and this inactivates p97/p47-mediated Golgi membrane fusion. Conversely, at the end of mitosis, VCIP135 is dephosphorylated (on Ser130), and this is associated with recovery of its deubiquitinase activity and with post-mitotic Golgi reassembly (Zhang and Wang, 2015). In addition to the stimulation of the fusion processes, dephosphorylation of the GRASP proteins favors the stacking and linking of the reformed Golgi cisternae, thus leading to the reassembly of the Golgi complex after mitosis.

In conclusion, the Golgi ribbon can be viewed as a metastable structure that is maintained by several core and accessory proteins, and that can rapidly adapt to stressful or physiological conditions.

\section{COORDINATION OF GOLGI FRAGMENTATION WITH THE CELL CYCLE}

\section{A. Why is the Severing of the Golgi Ribbon Necessary for G2/M Transition?}

It is now well-accepted that entry of cells into mitosis requires Golgi fragmentation (Sütterlin et al., 2002; Hidalgo Carcedo et al., 2004). Part of the signaling through which a block in Golgi fragmentation prevents cell-cycle progression has been identified. As the block of Golgi fragmentation induces G2 arrest, it is likely to involve cyclin-B-dependent kinase 1 (CycB-Cdk1), because $\mathrm{CycB}-\mathrm{Cdk} 1$ is the major regulator of $\mathrm{G} 2 / \mathrm{M}$ transition (Nigg, 2001). The activity of CycB1-Cdk1 activity is known to be controlled by recruitment and activation of the Ser/Thr kinase Aurora-A at the centrosome (Marumoto et al., 2002). Interestingly, a block in Golgi fragmentation interferes with Aurora-A recruitment to the centrosome in G2, and prevents its activation (Persico et al., 2010). This link between Golgi organization in G2 and Aurora-A recruitment and activation provides the first mechanistic insight into how Golgi dynamics can be coordinated with cell-cycle progression.

However, a second cyclinB-dependent, but Auroraindependent, mechanism might also contribute to Golgimediated control of mitotic entry. Mammalian cells express two B-type cyclins, $\mathrm{CycB} 1$ and $\mathrm{CycB} 2$, and $\mathrm{CycB} 2$ associates with the Golgi complex and the ER (Jackman et al., 1995). Both CycB1 and $\mathrm{CycB} 2$ cooperate to promote mitotic entry. It is possible that activation of the Golgi-associated CycB2-Cdk1 complex is regulated by inhibition of Golgi-localized Myt1 (Villeneuve et al., 2013). More investigations are required to address this issue.

\section{B. GTPases Control the Release of Golgi-Associated Proteins during Mitosis}

There is evidence that complete disassembly of the stacks is not required for mitotic progression (Uchiyama et al., 2003). Also, the second step of Golgi fragmentation in mammals (i.e., disassembly of isolated stacks) is involved in events that are necessary for cell-cycle progression. Indeed, during prophase, disassembly of the Golgi stacks correlates with release of Arf1 and a set of peripheral proteins from the Golgi membranes
(Altan-Bonnet et al., 2003). Arf GTPases are key regulators of membrane traffic and organelle structure, and they act through regulation of the recruitment of a large number of effectors. These include components of vesicular coats, membrane tethers, and lipid-binding or lipid-modifying enzymes (Cherfils, 2014). Spatial and temporal regulation of the Arf proteins is through their guanine-nucleotide-exchange factors (GEFs) and GTPaseactivating proteins (GAPs), which catalyze GTP binding and hydrolysis, respectively (Cherfils, 2014).

The mechanism through which Arf1 is released from Golgi membranes has started to emerge more recently. Indeed, a guanine nucleotide exchange factor for Arf that resides at the cis-Golgi, GBF1, is a key Arf regulator. During G2/M transition, GBF1 is phosphorylated by AMPK, which results in disassociation of GBF1 from Golgi membrane. Then during mitosis, GBF1 is also phosphorylated by CDK1. This results in the release of Arf from the Golgi membranes, but surprisingly, this does not cause the release of COPI, one of the main Arf effectors for membrane traffic (Morohashi et al., 2010). Phosphorylation of AMPK and GBF1 is essential for Golgi disassembly and subsequent mitosis entry. Active AMPK $\alpha$ (which is phosphorylated on Thr172) transiently localizes to the Golgi complex during late G2/early prophase. Functional inhibition of AMPK $\alpha$ delays G2/M transition in HeLa cells. Furthermore, it has been shown recently that $\mathrm{AMPK} \alpha 2$ is activated by CaMKK $\beta$-mediated phosphorylation during late G2 (Lee et al., 2015).

Golgin160 is among the key Golgi-located Arf effectors, and it recruits the dynein motor to regulate the position of the Golgi complex (Yadav et al., 2012). The minus-end-directed motor is required to confer centripetal motility to membranes. During cell division, the association of the dynein motor with membranes is regulated by dissociation of the receptor-motor complex from the membranes, which can explain the dramatic changes in organelle positioning observed during mitosis (Yadav et al., 2012).

An additional Golgi-located regulator of GTP-binding proteins is the non-catalytic $\beta$ subunit of RalGAP $\alpha 1 / 2 \beta$ (GTPase activator for RALA/B). RalGAP $\beta$ localizes to the Golgi complex and nucleus during interphase, and relocalizes to the mitotic spindle during mitosis. Depletion of RalGAP $\beta$ causes chromosome misalignment and decreases the levels of cyclin B1 (Personnic et al., 2014).

Proteins of the Rab family have also shown to have links to Golgi-based processes and mitotic progression. Rab6A' regulates retrograde transport from late endosomes via the Golgi to the $\mathrm{ER}$, and it is involved in the transition from metaphase to anaphase during mitosis. Bicaudal-D1 is an important effector of Rab6B (Wanschers et al., 2007). Rab6B and Bicaudal-D1 co-localize at the Golgi complex and on vesicles, where they regulate the association of these membranes with microtubules. Knocking down Rab6 $A^{\prime}$ reduces the endocytic intracellular transport of Shiga toxin and causes defects to Golgi-associated protein recycling through the ER. When the function of Rab6 $\mathrm{A}^{\prime}$ is altered, cells are blocked in metaphase with the Mad2-spindle checkpoint activated, which suggests that Rab6 $\mathrm{A}^{\prime}$ participates in a pathway that is involved in metaphase/anaphase transition 
(Miserey-Lenkei et al., 2006). Rab11 is localized at the transGolgi network and late endosomes during interphase. During mitosis, Rab11 localizes to the mitotic spindle and regulates dynein-dependent endosome localization at the mitotic spindle poles (Hehnly and Doxsey, 2014). Rab11 depletion prevents localization of recycling endosomes at the spindle poles, delays mitotic progression, disrupts spindle-pole protein recruitment and astral microtubule organization, and alters mitotic spindle orientation. This suggests that Rab11 is a crucial regulator of correct spindle-pole function during mitosis.

Altogether, these findings indicate that during mitosis, and concomitant with the disassembly of Golgi stacks, a series of GTP-binding proteins regulate the changes in the localization and function of Golgi-localized proteins, to regulate the key mitotic events. This suggests that Golgi disassembly is coordinated with chromosome segregation.

Related to this hypothesis, the Golgi-associated protein p115 has been shown to dissociate from the Golgi complex during mitosis. Instead, p115 partitions with the poles of the mitotic spindle, where it controls spindle formation and chromosome segregation and cytokinesis (Radulescu et al., 2011). Furthermore, dissociation of p115 upon mitotic entry favors the interaction of the golgin GM130 with importin $\alpha$, via a nuclearlocalization signal. This interaction leads to sequestration of importin $\alpha$ and liberates the spindle assembly factor TPX2, which can then activate Aurora-A kinase and stimulate local microtubule nucleation. At the spindle, microtubule nucleation is also favored by GM130, thus functionally linking Golgi membranes to spindle formation, which is crucial for faithful chromosome inheritance (Wei et al., 2015).

As a further example of a functional link between Golgi fragmentation and spindle formation, Miki is a Golgi-associated protein that is relocated during mitosis to the centrosome. This occurs through tankyrase-mediated PARsylation and controls the recruitment of CG-NAP, a scaffold protein of the $\gamma$-tubulin ring complex (Ozaki et al., 2012). Thus, Golgi vesiculation can be explained by the need to change the subcellular distribution of proteins that acquire specific and novel functions during mitosis.

\section{The Role of the Golgi Reformation during Telophase}

The Golgi membranes begin to reassemble during telophase, at which time they form two distinct ribbons on the opposite sides of the nucleus in each daughter cell (Altan-Bonnet et al., 2006; Goss and Toomre, 2008). The larger Golgi ribbon is positioned next to the centrosome, while the smaller one is in close proximity to the midbody. During cytokinesis, the smaller Golgi ribbon migrates to the opposite side of the nucleus and fuses with the larger Golgi ribbon. Overall these data suggest that a functional Golgi ribbon might be required to establish asymmetric cell division.

\section{CONCLUSIONS}

In conclusion, mitotic partitioning of the Golgi complex is composed of individual and consecutive steps that control different aspects of cell division (Figure 1). G2-specific fragmentation of the Golgi ribbon into isolated stacks is necessary for cell entry into mitosis. Then, during mitosis, selected Arf and Rab GTPases regulate the disassembly of the isolated stacks and the relocation of Golgi-associated proteins. This regulates the events that are necessary for successful cell duplication, such as spindle formation and chromosome segregation.

Further investigations into the Golgi fragmentation process and the molecular players involved in the control of the different stages of mitosis will contribute to better understanding of the mechanisms by which the disassembly of the Golgi complex controls G2/M transition and mitosis. These will have the potential to reveal novel targets and approaches for pharmacological intervention in many diseases, including cancers.

\section{AUTHOR CONTRIBUTIONS}

$\mathrm{CV}$ and $\mathrm{AC}$ wrote the manuscript.

\section{ACKNOWLEDGMENTS}

The authors would like to thank the Italian Association for Cancer Research (AIRC, Milan, Italy; IG6074 to AC), and MIURPON 01-00117 for financial support. The authors apologize to all colleagues whose work it has not been possible to cite in this review, due to space limitations. The authors also thank Chris Berrie for editorial assistance. PNR-CNR Aging Program 2012-2014 and Progetto Bandiera "Epigen."

\section{REFERENCES}

Acharya, U., Jacobs, R., Peters, J. M., Watson, N., Farquhar, M. G., and Malhotra, V. (1995). The formation of Golgi stacks from vesiculated Golgi membranes requires two distinct fusion events. Cell 82, 895-904. doi: 10.1016/00928674(95)90269-4

Acharya, U., Mallabiabarrena, A., Acharya, J. K., and Malhotra, V. (1998). Signaling via mitogen-activated protein kinase kinase (MEK1) is required for Golgi fragmentation during mitosis. Cell 92, 183-192. doi: 10.1016/S00928674(00)80913-7

Altan-Bonnet, N., Phair, R. D., Polishchuk, R. S., Weigert, R., and LippincottSchwartz, J. (2003). A role for Arf1 in mitotic Golgi disassembly, chromosome

segregation, and cytokinesis. Proc. Natl. Acad. Sci. U.S.A. 100, 13314-13319. doi: 10.1073/pnas.2234055100

Altan-Bonnet, N., Sougrat, R., and Lippincott-Schwartz, J. (2004). Molecular basis for Golgi maintenance and biogenesis. Curr. Opin. Cell Biol. 16, 364-372. doi: 10.1016/j.ceb.2004.06.011

Altan-Bonnet, N., Sougrat, R., Liu, W., Snapp, E. L., Ward, T., and LippincottSchwartz, J. (2006). Golgi inheritance in mammalian cells is mediated through endoplasmic reticulum export activities. Mol. Biol. Cell 17, 990-1005. doi: 10.1091/mbc.E05-02-0155

Axelsson, M. A., and Warren, G. (2004). Rapid, endoplasmic reticulumindependent diffusion of the mitotic Golgi haze. Mol. Biol. Cell 15, 1843-1852. doi: 10.1091/mbc.E03-07-0459 
Barr, F. A. (2004). Golgi inheritance: shaken but not stirred. J. Cell Biol. 164, 955-958. doi: 10.1083/jcb.200402011

Beams, H. W., and Kessel, R. G. (1968). The Golgi apparatus: structure and function. Int. Rev. Cytol. 23, 209-276. doi: 10.1016/S0074-7696(08)60273-9

Brunger, A. T., and DeLaBarre, B. (2003). NSF and p97/VCP: similar at first, different at last. FEBS Lett. 555, 126-133. doi: 10.1016/S0014-5793(03)01107-4

Cervigni, R. I., Bonavita, R., Barretta, M. L., Spano, D., Ayala, I., Nakamura, N., et al. (2015). JNK2 controls fragmentation of the Golgi complex and the G2/M transition through phosphorylation of GRASP65. J. Cell Sci. 128, 2249-2260. doi: $10.1242 /$ jcs. 164871

Cherfils, J. (2014). Arf GTPases and their effectors: assembling multivalent membrane-binding platforms. Curr. Opin. Struct. Biol. 29, 67-76. doi: 10.1016/j.sbi.2014.09.007

Colanzi, A., and Corda, D. (2007). Mitosis controls the Golgi and the Golgi controls mitosis. Curr. Opin. Cell Biol. 19, 386-393. doi: 10.1016/j.ceb.2007.06.002

Colanzi, A., Hidalgo Carcedo, C., Persico, A., Cericola, C., Turacchio, G., Bonazzi, M., et al. (2007). The Golgi mitotic checkpoint is controlled by BARSdependent fission of the Golgi ribbon into separate stacks in G2. EMBO J. 26, 2465-2476. doi: $10.1038 /$ sj.emboj. 7601686

Colanzi, A., and Sütterlin, C. (2013). Signaling at the Golgi during mitosis. Methods Cell Biol. 118, 383-400. doi: 10.1016/B978-0-12-417164-0.00023-9

Colanzi, A., Sutterlin, C., and Malhotra, V. (2003). RAF1-activated MEK1 is found on the Golgi apparatus in late prophase and is required for Golgi complex fragmentation in mitosis. J. Cell Biol. 161, 27-32. doi: 10.1083/jcb.200208099

Cornwell, W. D., Kaminski, P. J., and Jackson, J. R. (2002). Identification of Drosophila Myt1 kinase and its role in Golgi during mitosis. Cell Signal. 14, 467-476. doi: 10.1016/S0898-6568(01)00276-5

De Matteis, M. A., and Luini, A. (2008). Exiting the Golgi complex. Nat. Rev. Mol. Cell Biol. 9, 273-284. doi: 10.1038/nrm2378

Duran, J. M., Kinseth, M., Bossard, C., Rose, D. W., Polishchuk, R., Wu, C. C., et al. (2008). The role of GRASP55 in Golgi fragmentation and entry of cells into mitosis. Mol. Biol. Cell 19, 2579-2587. doi: 10.1091/mbc.E07-10-0998

Feinstein, T. N., and Linstedt, A. D. (2007). Mitogen-activated protein kinase kinase 1-dependent Golgi unlinking occurs in G2 phase and promotes the G2/M cell cycle transition. Mol. Biol. Cell 18, 594-604. doi: 10.1091/mbc.E0606-0530

Feinstein, T. N., and Linstedt, A. D. (2008). GRASP55 regulates Golgi ribbon formation. Mol. Biol. Cell 19, 2696-2707. doi: 10.1091/mbc.E07-11-1200

Goss, J. W., and Toomre, D. K. (2008). Both daughter cells traffic and exocytose membrane at the cleavage furrow during mammalian cytokinesis. J. Cell Biol. 181, 1047-1054. doi: 10.1083/jcb.200712137

Hehnly, H., and Doxsey, S. (2014). Rab11 endosomes contribute to mitotic spindle organization and orientation. Dev. Cell 28, 497-507. doi: 10.1016/j.devcel.2014.01.014

Hidalgo Carcedo, C., Bonazzi, M., Spanó, S., Turacchio, G., Colanzi, A., Luini, A., et al. (2004). Mitotic Golgi partitioning is driven by the membrane-fissioning protein CtBP3/BARS. Science 305, 93-96. doi: 10.1126/science.1097775

Jackman, M., Firth, M., and Pines, J. (1995). Human cyclins B1 and B2 are localized to strikingly different structures: B1 to microtubules, B2 primarily to the Golgi apparatus. EMBO J. 14, 1646-1654.

Jarvela, T., and Linstedt, A. D. (2014). Isoform-specific tethering links the Golgi ribbon to maintain compartmentalization. Mol. Biol. Cell 25, 133-144. doi: 10.1091/mbc.E13-07-0395

Kaneko, Y., Tamura, K., Totsukawa, G., and Kondo, H. (2010). Phosphorylation of p37 is important for Golgi disassembly at mitosis. Biochem. Biophys. Res. Commun. 402, 37-41. doi: 10.1016/j.bbrc.2010.09.097

Kano, F., Tanaka, A. R., Yamauchi, S., Kondo, H., and Murata, M. (2004). Cdc2 kinase-dependent disassembly of endoplasmic reticulum (ER) exit sites inhibits ER-to-Golgi vesicular transport during mitosis. Mol. Biol. Cell 15, 4289-4298. doi: 10.1091/mbc.E03-11-0822

Kienzle, C., Eisler, S. A., Villeneuve, J., Brummer, T., Olayioye, M. A., and Hausser, A. (2013). PKD controls mitotic Golgi complex fragmentation through a Raf-MEK1 pathway. Mol. Biol. Cell 24, 222-233. doi: 10.1091/mbc.E1203-0198

Kodani, A., and Sütterlin, C. (2009). A new function for an old organelle: microtubule nucleation at the Golgi apparatus. EMBO J. 28, 995-996. doi: 10.1038/emboj.2009.85
Kondo, H., Rabouille, C., Newman, R., Levine, T. P., Pappin, D., Freemont, P., et al. (1997). p47 is a cofactor for p97-mediated membrane fusion. Nature 388, 75-78. doi: 10.1038/40411

Lee, I. J., Lee, C. W., and Lee, J. H. (2015). CaMKKbeta-AMPKalpha2 signaling contributes to mitotic Golgi fragmentation and the G2/M transition in mammalian cells. Cell Cycle 14, 598-611. doi: 10.4161/15384101.2014.991557

Liberali, P., Kakkonen, E., Turacchio, G., Valente, C., Spaar, A., Perinetti, G., et al. (2008). The closure of Pak1-dependent macropinosomes requires the phosphorylation of CtBP1/BARS. EMBO J. 27, 970-981. doi: 10.1038/emboj.2008.59

Lin, C. Y., Madsen, M. L., Yarm, F. R., Jang, Y. J., Liu, X., and Erikson, R. L. (2000). Peripheral Golgi protein GRASP65 is a target of mitotic polo-like kinase (Plk) and Cdc2. Proc. Natl. Acad. Sci. U.S.A 97, 12589-12594. doi: 10.1073/pnas.220423497

Liu, J., Flanagan, W. M., Drazba, J. A., Estes, M. L., Barnett, G. H., Haqqi, T., et al. (1997). The CDK inhibitor, p27Kip1, is required for IL-4 regulation of astrocyte proliferation. J. Immunol. 159, 812-819.

López-Sánchez, I., Sanz-García, M., and Lazo, P. A. (2009). Plk3 interacts with and specifically phosphorylates VRK1 in Ser342, a downstream target in a pathway that induces Golgi fragmentation. Mol. Cell Biol. 29, 1189-1201. doi: 10.1128/MCB.01341-08

Marra, P., Salvatore, L., Mironov, A. Jr., Di Campli, A., Di Tullio, G., Trucco, A., et al. (2007). The biogenesis of the Golgi ribbon: the roles of membrane input from the ER and of GM130. Mol. Biol. Cell 18, 1595-1608. doi: 10.1091/mbc.E06-10-0886

Marumoto, T., Hirota, T., Morisaki, T., Kunitoku, N., Zhang, D., Ichikawa, Y., et al. (2002). Roles of aurora-A kinase in mitotic entry and G2 checkpoint in mammalian cells. Genes Cells 7, 1173-1182. doi: 10.1046/j.13652443.2002.00592.x

Meyer, H. H., Shorter, J. G., Seemann, J., Pappin, D., and Warren, G. (2000). A complex of mammalian ufd 1 and npl4 links the AAA-ATPase, p97, to ubiquitin and nuclear transport pathways. EMBO J. 19, 2181-2192. doi: 10.1093/emboj/19.10.2181

Miserey-Lenkei, S., Couëdel-Courteille, A., Del Nery, E., Bardin, S., Piel, M., Racine, V., et al. (2006). A role for the Rab6A' GTPase in the inactivation of the Mad2-spindle checkpoint. EMBO J. 25, 278-289. doi: 10.1038/sj.emboj.7600929

Misteli, T., and Warren, G. (1995). Mitotic disassembly of the Golgi apparatus in vivo. J. Cell Sci. 108(Pt 7), 2715-2727.

Mogelsvang, S., Gomez-Ospina, N., Soderholm, J., Glick, B. S., and Staehelin, L. A. (2003). Tomographic evidence for continuous turnover of Golgi cisternae in Pichia pastoris. Mol. Biol. Cell 14, 2277-2291. doi: 10.1091/mbc.E02-10-0697

Morohashi, Y., Balklava, Z., Ball, M., Hughes, H., and Lowe, M. (2010). Phosphorylation and membrane dissociation of the ARF exchange factor GBF1 in mitosis. Biochem. J. 427, 401-412. doi: 10.1042/BJ20091681

Munro, S. (2011). The golgin coiled-coil proteins of the Golgi apparatus. Cold Spring Harb. Perspect. Biol. 3:a005256. doi: 10.1101/cshperspect.a005256

Nakajima, H., Yonemura, S., Murata, M., Nakamura, N., Piwnica-Worms, H., and Nishida, E. (2008). Myt1 protein kinase is essential for Golgi and ER assembly during mitotic exit. J. Cell Biol. 181, 89-103. doi: 10.1083/jcb.200708176

Nigg, E. A. (2001). Mitotic kinases as regulators of cell division and its checkpoints. Nat. Rev. Mol. Cell Biol. 2, 21-32. doi: 10.1038/35048096

Ozaki, Y., Matsui, H., Asou, H., Nagamachi, A., Aki, D., Honda, H., et al. (2012). Poly-ADP ribosylation of Miki by tankyrase-1 promotes centrosome maturation. Mol. Cell 47, 694-706. doi: 10.1016/j.molcel.2012.06.033

Papanikou, E., and Glick, B. S. (2009). The yeast Golgi apparatus: insights and mysteries. FEBS Lett. 583, 3746-3751. doi: 10.1016/j.febslet.2009.10.072

Persico, A., Cervigni, R. I., Barretta, M. L., Corda, D., and Colanzi, A. (2010). Golgi partitioning controls mitotic entry through Aurora-A kinase. Mol. Biol. Cell 21, 3708-3721. doi: 10.1091/mbc.E10-03-0243

Personnic, N., Lakisic, G., Gouin, E., Rousseau, A., Gautreau, A., Cossart, P., et al. (2014). A role for Ral GTPase-activating protein subunit beta in mitotic regulation. FEBS J. 281, 2977-2989. doi: 10.1111/febs.12836

Preisinger, C., Körner, R., Wind, M., Lehmann, W. D., Kopajtich, R., and Barr, F. A. (2005). Plk1 docking to GRASP65 phosphorylated by Cdk1 suggests a mechanism for Golgi checkpoint signalling. EMBO J. 24, 753-765. doi: 10.1038/sj.emboj.7600569 
Prescott, A. R., Farmaki, T., Thomson, C., James, J., Paccaud, J. P., Tang, B. L., et al. (2001). Evidence for prebudding arrest of ER export in animal cell mitosis and its role in generating Golgi partitioning intermediates. Traffic 2, 321-335. doi: 10.1034/j.1600-0854.2001.002005321.x

Puri, S., Telfer, H., Velliste, M., Murphy, R. F., and Linstedt, A. D. (2004). Dispersal of Golgi matrix proteins during mitotic Golgi disassembly. J. Cell Sci. 117, 451-456. doi: 10.1242/jcs.00863

Puthenveedu, M. A., Bachert, C., Puri, S., Lanni, F., and Linstedt, A. D. (2006). GM130 and GRASP65-dependent lateral cisternal fusion allows uniform Golgienzyme distribution. Nat. Cell Biol. 8, 238-248. doi: 10.1038/ncb1366

Rabouille, C., Levine, T. P., Peters, J. M., and Warren, G. (1995). An NSFlike ATPase, p97, and NSF mediate cisternal regrowth from mitotic Golgi fragments. Cell 82, 905-914. doi: 10.1016/0092-8674(95)90270-8

Radulescu, A. E., Mukherjee, S., and Shields, D. (2011). The Golgi protein p115 associates with gamma-tubulin and plays a role in Golgi structure and mitosis progression. J. Biol. Chem. 286, 21915-21926. doi: 10.1074/jbc.M110.209460

Rambourg, A., and Clermont, Y. (1990). Three-dimensional electron microscopy: structure of the Golgi apparatus. Eur. J. Cell Biol. 51, 189-200.

Rhee, S. W., Starr, T., Forsten-Williams, K., and Storrie, B. (2005). The steadystate distribution of glycosyltransferases between the Golgi apparatus and the endoplasmic reticulum is approximately 90:10. Traffic 6, 978-990. doi: 10.1111/j.1600-0854.2005.00333.x

Rios, R. M., and Bornens, M. (2003). The Golgi apparatus at the cell centre. Curr. Opin. Cell Biol. 15, 60-66. doi: 10.1016/S0955-0674(02)00013-3

Rossanese, O. W., Soderholm, J., Bevis, B. J., Sears, I. B., O’Connor, J., Williamson, E. K., et al. (1999). Golgi structure correlates with transitional endoplasmic reticulum organization in Pichia pastoris and Saccharomyces cerevisiae. J. Cell Biol. 145, 69-81. doi: 10.1083/jcb.145.1.69

Rothman, J. E., and Wieland, F. T. (1996). Protein sorting by transport vesicles. Science 272, 227-234. doi: 10.1126/science.272.5259.227

Ruan, Q., Wang, Q., Xie, S., Fang, Y., Darzynkiewicz, Z., Guan, K., et al. (2004). Polo-like kinase 3 is Golgi localized and involved in regulating Golgi fragmentation during the cell cycle. Exp. Cell Res. 294, 51-59. doi: 10.1016/j.yexcr.2003.10.022

Seemann, J., Pypaert, M., Taguchi, T., Malsam, J., and Warren, G. (2002). Partitioning of the matrix fraction of the Golgi apparatus during mitosis in animal cells. Science 295, 848-851. doi: 10.1126/science.1068064

Sengupta, D., and Linstedt, A. D. (2010). Mitotic inhibition of GRASP65 organelle tethering involves Polo-like kinase 1 (PLK1) phosphorylation proximate to an internal PDZ ligand. J. Biol. Chem. 285, 39994-40003. doi: 10.1074/jbc.M110.189449

Shaul, Y. D., and Seger, R. (2006). ERK1c regulates Golgi fragmentation during mitosis. J. Cell Biol. 172, 885-897. doi: 10.1083/jcb.200509063

Shima, D. T., Cabrera-Poch, N., Pepperkok, R., and Warren, G. (1998). An ordered inheritance strategy for the Golgi apparatus: visualization of mitotic disassembly reveals a role for the mitotic spindle. J. Cell Biol. 141, 955-966. doi: $10.1083 /$ jcb.141.4.955

Shorter, J., and Warren, G. (2002). Golgi architecture and inheritance. Annu. Rev. Cell Dev. Biol. 18, 379-420. doi: 10.1146/annurev.cellbio.18.030602.133733

Sönnichsen, B., Watson, R., Clausen, H., Misteli, T., and Warren, G. (1996). Sorting by COP I-coated vesicles under interphase and mitotic conditions. J. Cell Biol. 134, 1411-1425. doi: 10.1083/jcb.134.6.1411

Sütterlin, C., and Colanzi, A. (2010). The Golgi and the centrosome: building a functional partnership. J. Cell Biol. 188, 621-628. doi: 10.1083/jcb.200910001

Sütterlin, C., Hsu, P., Mallabiabarrena, A., and Malhotra, V. (2002). Fragmentation and dispersal of the pericentriolar Golgi complex is required for entry into mitosis in mammalian cells. Cell 109, 359-369. doi: 10.1016/S00928674(02)00720-1

Sütterlin, C., Lin, C. Y., Feng, Y., Ferris, D. K., Erikson, R. L., and Malhotra, V. (2001). Polo-like kinase is required for the fragmentation of pericentriolar Golgi stacks during mitosis. Proc. Natl. Acad. Sci. U.S.A. 98, 9128-9132. doi: 10.1073/pnas. 161283998

Sütterlin, C., Polishchuk, R., Pecot, M., and Malhotra, V. (2005). The Golgiassociated protein GRASP65 regulates spindle dynamics and is essential for cell division. Mol. Biol. Cell 16, 3211-3222. doi: 10.1091/mbc.E04-12-1065

Tang, D., Yuan, H., and Wang, Y. (2010). The role of GRASP65 in Golgi cisternal stacking and cell cycle progression. Traffic 11, 827-842. doi: 10.1111/j.16000854.2010.01055.x
Thyberg, J., and Moskalewski, S. (1999). Role of microtubules in the organization of the Golgi complex. Exp. Cell Res. 246, 263-279. doi: 10.1006/excr.1998.4326

Totsukawa, G., Matsuo, A., Kubota, A., Taguchi, Y., and Kondo, H. (2013). Mitotic phosphorylation of VCIP135 blocks p97ATPase-mediated Golgi membrane fusion. Biochem. Biophys. Res. Commun. 433, 237-242. doi: 10.1016/j.bbrc.2013.02.090

Truschel, S. T., Zhang, M., Bachert, C., Macbeth, M. R., and Linstedt, A. D. (2012). Allosteric regulation of GRASP protein-dependent Golgi membrane tethering by mitotic phosphorylation. J. Biol. Chem. 287, 19870-19875. doi: 10.1074/jbc.M111.326256

Uchiyama, K., Jokitalo, E., Kano, F., Murata, M., Zhang, X., Canas, B., et al. (2002). VCIP135, a novel essential factor for p97/p47-mediated membrane fusion, is required for Golgi and ER assembly in vivo. J. Cell Biol. 159, 855-866. doi: $10.1083 /$ jcb. 200208112

Uchiyama, K., Jokitalo, E., Lindman, M., Jackman, M., Kano, F., Murata, M., et al. (2003). The localization and phosphorylation of p47 are important for Golgi disassembly-assembly during the cell cycle. J. Cell Biol. 161, 1067-1079. doi: $10.1083 /$ jcb. 200303048

Uchiyama, K., Totsukawa, G., Puhka, M., Kaneko, Y., Jokitalo, E., Dreveny, I., et al. (2006). p37 is a p97 adaptor required for Golgi and ER biogenesis in interphase and at the end of mitosis. Dev. Cell 11, 803-816. doi: 10.1016/j.devcel.2006.10.016

Valente, C., Luini, A., and Corda, D. (2013). Components of the CtBP1/BARSdependent fission machinery. Histochem. Cell Biol. 140, 407-421. doi: 10.1007/s00418-013-1138-1

Valente, C., Turacchio, G., Mariggió, S., Pagliuso, A., Gaibisso, R., Di Tullio, G., et al. (2012). A 14-3-3gamma dimer-based scaffold bridges CtBP1-S/BARS to PI(4)KIIIbeta to regulate post-Golgi carrier formation. Nat. Cell Biol. 14, 343-354. doi: 10.1038/ncb2445

Villeneuve, J., Scarpa, M., Ortega-Bellido, M., and Malhotra, V. (2013). MEK1 inactivates Myt1 to regulate Golgi membrane fragmentation and mitotic entry in mammalian cells. EMBO J. 32, 72-85. doi: 10.1038/emboj.2012.329

Vinke, F. P., Grieve, A. G., and Rabouille, C. (2011). The multiple facets of the Golgi reassembly stacking proteins. Biochem. J. 433, 423-433. doi: 10.1042/BJ20101540

Wang, Y., Satoh, A., and Warren, G. (2005). Mapping the functional domains of the Golgi stacking factor GRASP65. J. Biol. Chem. 280, 4921-4928. doi: 10.1074/jbc.M412407200

Wang, Y., Seemann, J., Pypaert, M., Shorter, J., and Warren, G. (2003). A direct role for GRASP65 as a mitotically regulated Golgi stacking factor. EMBO J. 22, 3279-3290. doi: 10.1093/emboj/cdg317

Wanschers, B. F., van de Vorstenbosch, R., Schlager, M. A., Splinter, D., Akhmanova, A., Hoogenraad, C. C., et al. (2007). A role for the Rab6B BicaudalD1 interaction in retrograde transport in neuronal cells. Exp. Cell Res. 313, 3408-3420. doi: 10.1016/j.yexcr.2007.05.032

Wei, J. H., and Seemann, J. (2009). Spindle-dependent partitioning of the Golgi ribbon. Commun. Integr. Biol. 2, 406-407. doi: 10.4161/cib.2.5.8764

Wei, J. H., Zhang, Z. C., Wynn, R. M., and Seemann, J. (2015). GM130 Regulates Golgi-derived spindle assembly by activating TPX2 and capturing microtubules. Cell 162, 287-299. doi: 10.1016/j.cell.2015.06.014

Xiang, Y., and Wang, Y. (2010). GRASP55 and GRASP65 play complementary and essential roles in Golgi cisternal stacking. J. Cell Biol. 188, 237-251. doi: $10.1083 /$ jcb. 200907132

Xiang, Y., Zhang, X., Nix, D. B., Katoh, T., Aoki, K., Tiemeyer, M., et al. (2013). Regulation of protein glycosylation and sorting by the Golgi matrix proteins GRASP55/65. Nat. Commun. 4, 1659. doi: 10.1038/ncomms2669

Xie, S., Wang, Q., Ruan, Q., Liu, T., Jhanwar-Uniyal, M., Guan, K., et al. (2004). MEK1-induced Golgi dynamics during cell cycle progression is partly mediated by Polo-like kinase-3. Oncogene 23, 3822-3829. doi: 10.1038/sj.onc.1207479

Yadav, S., Puri, S., and Linstedt, A. D. (2009). A primary role for Golgi positioning in directed secretion, cell polarity, and wound healing. Mol. Biol. Cell 20, 1728-1736. doi: 10.1091/mbc.E08-10-1077

Yadav, S., Puthenveedu, M. A., and Linstedt, A. D. (2012). Golgin160 recruits the dynein motor to position the Golgi apparatus. Dev. Cell 23, 153-165. doi: 10.1016/j.devcel.2012.05.023

Yeaman, C., Ayala, M. I., Wright, J. R., Bard, F., Bossard, C., Ang, A., et al. (2004). Protein kinase $\mathrm{D}$ regulates basolateral membrane protein exit from trans-Golgi network. Nat. Cell Biol. 6, 106-112. doi: 10.1038/ncb1090 
Yoshimura, S., Yoshioka, K., Barr, F. A., Lowe, M., Nakayama, K., Ohkuma, S., et al. (2005). Convergence of cell cycle regulation and growth factor signals on GRASP65. J. Biol. Chem. 280, 23048-23056. doi: 10.1074/jbc.M5024 42200

Zaal, K. J., Smith, C. L., Polishchuk, R. S., Altan, N., Cole, N. B., Ellenberg, J., et al. (1999). Golgi membranes are absorbed into and reemerge from the ER during mitosis. Cell 99, 589-601. doi: 10.1016/S0092-8674(00)81548-2

Zhang, X., and Wang, Y. (2015). Cell cycle regulation of VCIP135 deubiquitinase activity and function in p97/p47-mediated Golgi reassembly. Mol. Biol. Cell 26, 2242-2251. doi: 10.1091/mbc.E15-01-0041
Conflict of Interest Statement: The authors declare that the research was conducted in the absence of any commercial or financial relationships that could be construed as a potential conflict of interest.

Copyright (๑) 2015 Valente and Colanzi. This is an open-access article distributed under the terms of the Creative Commons Attribution License (CC BY). The use, distribution or reproduction in other forums is permitted, provided the original author(s) or licensor are credited and that the original publication in this journal is cited, in accordance with accepted academic practice. No use, distribution or reproduction is permitted which does not comply with these terms. 